EPJ Web of Conferences 61, 09001 (2013)

DOI: $10.1051 /$ epjconf/ 20136109001

(C) Owned by the authors, published by EDP Sciences, 2013

\title{
The Innermost Regions of Relativistic Jets: Wrapping Up the Enigma
}

\author{
Alan P. Marscher ${ }^{1}$ \\ ${ }^{1}$ Institute for Astrophysical Research, Boston University, 725 Commonwealth Ave., Boston, MA 02215 USA e-mail: marscher@ \\ bu.edu
}

\begin{abstract}
What are relativistic jets like within a million Schwarzschild radii of the accreting black hole that powers them? A meeting in Granada, Spain in June 2013, organized by José L. Gómez and his conspirators brought together observers and theorists to survey the current state of observational data and efforts to interpret them. This conference summary reviews the results, insights, arguments, conflicts, and agreements that occurred during five sunny days spent in a windowless room in a hotel at the bottom of the hill that holds the heart of the beautiful city.
\end{abstract}

\section{Introduction}

Active galactic nuclei (AGN) present us with many of the most puzzling mysteries of the cosmos. Their most extreme subclass, blazars, are the most luminous objects in the universe, yet are powered by solar-system-sized supermassive black holes. Black holes are also at the hearts of their little cousins, high-mass X-ray binary systems (XRBs). The basic process that powers blazars is accretion onto the black hole, yet most of the luminosity is produced by relativistic magnetized plasma jets that are propelled along the rotational poles of the system. Particles in the jets need to be accelerated to energies up to the $\mathrm{TeV}$ range in order to produce the radiation that we see across the electromagnetic spectrum. This process must be extremely efficient, since blazars are such bright $\gamma$-ray emitters that they dominate the $\gamma$-ray sky outside of the plane of the Milky Way. Nature seems to find this very easy to accomplish the launching of jets and energization of its particles, yet we struggle to understand how it manages to do so.

In a blazar, we have the great fortune to be viewing one of the jets nearly right down the axis, which allows us to view all of the glories of the near-light velocities. This includes the illusion of superluminal motion, blueshifting and beaming of the radiation, and shortened time-scales of variability. While we need to figure out how to deal with the illusions in order to figure out how blazars work, the beaming at least allows us to view the jet without confusing its radiation with that of the thermal components, except over relatively narrow ranges of frequencies (mostly optical and UV) in the case of quasars.

In the case of XRBs, the jet is usually at a wider angle to the observer. Because of this, it is not so clear whether emission at a given waveband is from the jet or the accretion disk/corona complex. But things happen fast in $\mathrm{XRBs}$, so that we can observe how conditions change as the system generates continuously flowing jets, sends major disturbances down the jets, and shuts the jets off.

The conference concentrated on progress that we have made in gathering comprehensive datasets of blazar emission and in relating the data to the physical conditions and processes that occur in the jets. While all of the attendees sought to unwrap the enigma ${ }^{2}$ of blazars, many vexing puzzles remain. I have been assigned the task of summarizing the presentations and discussions that took place at the conference. It would take me many more pages than the reader would want to wade through, and an inordinate amount of time in writing, to perform this task with the diligence that the undertaking deserves. Because of this, I can only assert that this summary reflects some of the highlights of the meeting and covers a non-neglible fraction of the fine work that was presented there.

\section{Overview of the Conference}

As illustrated in Figure 1, the meeting blended the two rather distinct universes of theory and observations. Many of the theorists who create simulated jets from first principles (as opposed to modelers such as I, who assume that the jet already exists and give it some $a$ priori properties in order to calculate its behavior) are now paying close attention to the observational images, etc., in an attempt to connect their fantasies with reality. This is greatly appreciated by many observers. Others are applying techniques of theory to direct interpretation of observations. In fact, one hard-core observer, Denise Gabuzda, made the startling declaration "Monte Carlo simulations don't lie,"

\footnotetext{
${ }^{2}$ The second half of the title of this conference summary twists the words of the late Winston Churchill regarding his expressed inability to predict the actions of Russia during the early stages of World War II. We certainly have no less uncertainty in our ability to predict what will happen next in any of the blazars we study!
} 


\section{Two Universes Connected by Jets Joined by this Meeting}

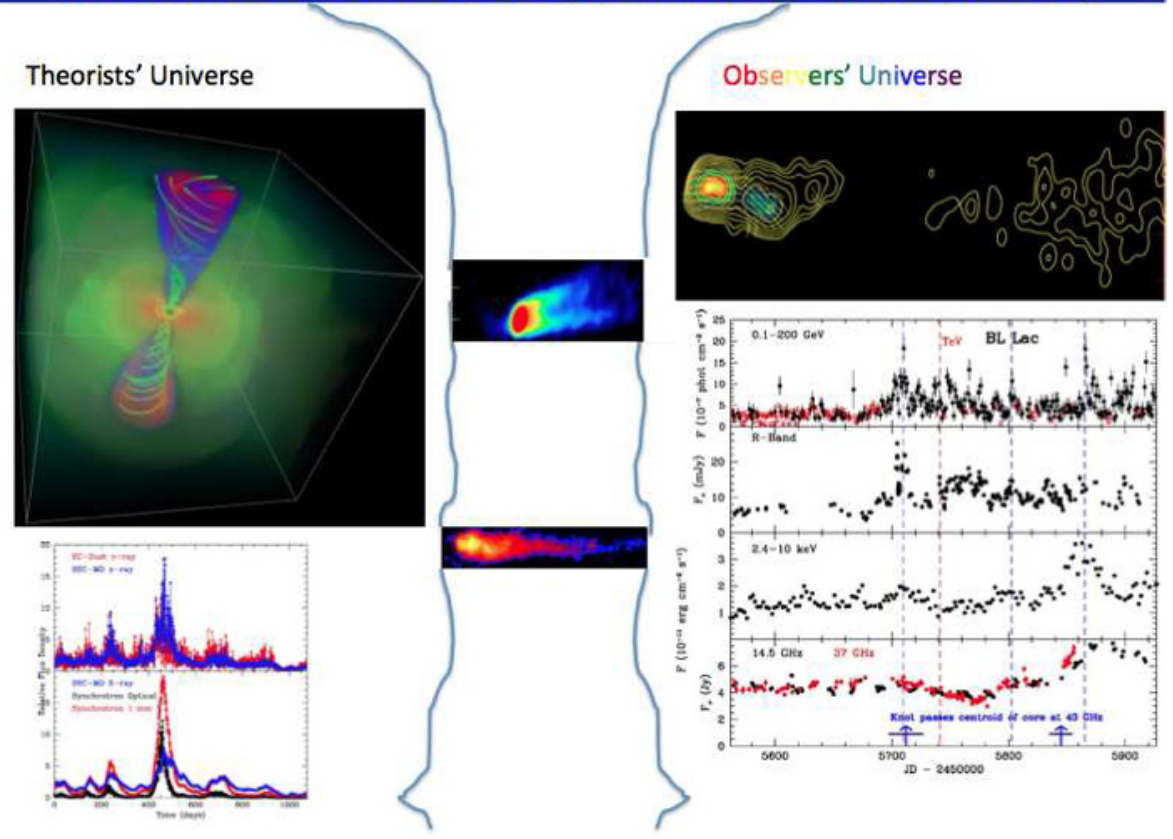

Figure 1. Two universes were joined by the conference, with jets such as the one in M87 acting as wormholes. Images in the middle are from the NRAO image gallery.

words that I never thought would be uttered by someone so well-grounded in data.

\section{Creation, Stability, and Dynamics of Jets}

Figure 2 depicts a jet created by black magic. Playing the part of the sorceress at the meeting was Sasha Tchekhovskoy, who gave a review of numerical studies of jet production by accreting black holes. I was impressed that it took about one year of computing time to run his code for the case of a MAD (magnetically-arrested accretion disk) scenario, in which the flow writhed in anguish as it gave birth to a pair of jets. Ioannis Contopoulos opined, as a caution against thinking that we have solved all of the problems related to the creation of jets: "I think that we are moving too fast with numerical simulations." If it takes one year to make a single jet, I don't think so!

A simple calculation reveals something very interesting about Sasha's computation. The year-long run corresponded to a time in the simulated system of $\Delta t=$ $3 \times 10^{4} R_{g} / c$, where $R_{g}$ is the gravitational radius of the black hole $(\mathrm{BH})$. For a $\mathrm{BH}$ of mass $10^{8} M_{\odot}$, this means that the code took 4.3 times as long to run as the time it was simulating. The time dilation factor is then 4.3 , so that the computer must have been at $r=1.057 R_{s}$ ! Sasha never told us how he managed to extract the output from so close to the event horizon.

Various presenters at the meeting, whose names are given in Figure 2, discussed kinks and instabilities that occur farther downstream in the jet. From this and their basic phallic shape, I conclude that jets are very, very sexy, and often kinky. Therefore, we need a more provocative name for them. I suggest Magnado del Diablo, a shortened version of "magnetic tornado of the devil." And who is the devil? At the meeting, Dr. Tchekhovskoy played the part very well!

Some of the theorists at the conference discussed matters that jet simulators should consider. These include return currents (Ioannis Contopoulos), geometrically thick accretion disks (Xiang Liu), tilted disks (Danilo Texeira), and binary black holes (Gabriela Vila). John Kirk discussed how a Poynting-dominated flow turns into particleenergy-dominated plasma via waves in MHD shocks. The plasma then emits synchro-Compton radiation with polarization electric vector transverse to the jet axis. Quasars often have this polarization direction, so we need to consider the process in our interpretations.

Among the latest insights on the general properties of jets provided at the meeting include:

- 3D MHD simulations indicate that more open field lines produce somewhat slower jets (Jan Staff).

- Magnetic collimation in M87 fits the data better than does a conical jet model (Masanori Nakamura).

- The jets of X-ray binaries (XRBs) turn on and off (Sera Markoff).

- Thicker disks make less powerful jets from the Blandford-Payne mechanism in XRBs (Jonathan Ferreira).

- An AGN jet can arise suddenly, as is thought to have occurred during a tidal disruption event that created the “instant blazar" Swift J1644+57 (Alberto Castro-Tirado). - If a cloud or wind enters a jet, shocks and other phenomena can result, leading to emission features (Valenti 

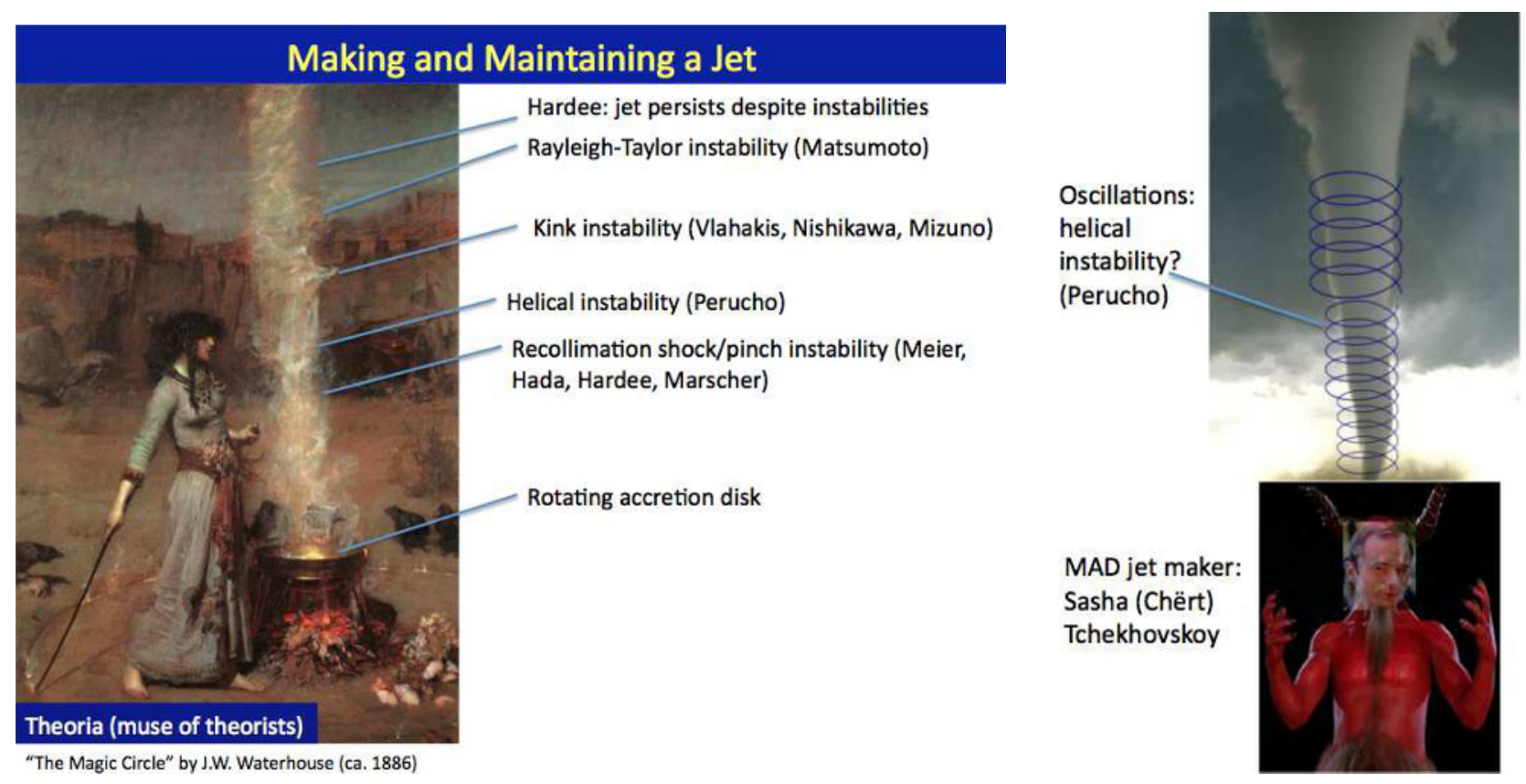

Figure 2. Left: Sorcerers, as well as nature, knew how to make jets long before theorists figured it out, but they lacked the ability to analyze all of a jet's features that theorists attending the meeting demonstrated. Right: Now theorists are able to create jets in their computers, as does MAD scientist Sasha Tchekhovskoy, depicted here as a chërt (Russian word for "devil"). Manel Perucho is no devil, but it appears that he is rather unstable, since he has a preference for instabilities in jets.

\section{Bosch-Ramon).}

One of the main questions regarding jets is whether they are dominated by Poynting flux or kinetic energy. Most theorists would say that the magnetic field dominates the energy density within $10^{4}$ to $10^{6}$ gravitational radii, beyond which kinetic flux takes over. Dave Meier proposed that FR I sources remain Poynting flux dominated beyond the main jet acceleration and collimation zone, while FR II objects become kinetic flux dominated. In support of this, Matt Lister reported that, based on the MOJAVE survey with the VLBA, apparent speeds of BL Lac objects tend to increase with distance from the "core," whereas this is not so common in quasars. John Wardle affirmed the standard result that the electric vectors of the linear polarization of BL Lac objects tend to align with the jet direction, consistent with the helical field expected when Poynting flux dominates. Quasar polarization vectors show no trend on parsec scales (but tend to be transverse to the jet on kpc scales). Dave Meier predicted that BL Lac jets should show signs of non-ballistic motions and kinks, and Marshall Cohen found a prominent example in BL Lacertae. On the other hand, there is no shortage of these effects in quasars and BL Lacs, so the test may not be a robust way to discern differences in the energy content of the jets of the two classes.

\section{Sites of Flares in Jets}

We rely on time variations of the emission from jets to probe the physics behind their extravagent activities. In order to accomplish this, we need to know where the variations are occurring. But blazars are too capricious and coy to reveal their secrets readily. Time-scales of variability of minutes or hours involving a substantial fraction of the nonthermal luminosity suggest that flares occur within light-days of the black hole, as championed at the meeting by Gabriele Ghisellini. His arguments extend beyond the rapid fluctuations in brightness. This scenario provides ample sources of seed photons for inverse Compton scattering to produce $\mathrm{X}$-rays and $\gamma$-rays, with the accretion disk, broad emission-line region (BLR), and dusty molecular torus all possible contributors. The X-ray to $\gamma$ ray spectral energy distributions (SEDs) of blazars whose luminosities are dominated by $\gamma$-ray emission are wellreproduced if the spectrum of seed photons is thermal in nature, as for these three sources. Also, the $\gamma$-ray spectra of some blazars appear to have a sharp break at $2 \mathrm{GeV}$, which can be explained by opacity to pair production off helium line photons.

To counter these attractions, timing of the flares at different wavebands and changes in the parsec-scale jet structure - which are simultaneous or with the latter occurring first in many cases, as shown by Iván Agudo, Svetlana Jorstad, Mikhail Lisakov, Hiroshi Nagai, and others at the meeting - indicate that most of the action occurs on parsec scales. This is supported by the insistence of some quasars to allow sub- $\mathrm{TeV} \gamma$-ray photons to escape in our direction, showing us that they can escape the prison of optical photons that would force them to be executed by producing electron-positron pairs. Luigi Pacciani and Bagmeet Behera discussed this issue, which is particularly acute in the quasar $1222+216$, from which sub- $\mathrm{TeV}$ photons are seen to vary on time-scales as short as $\sim 20$ minutes. 

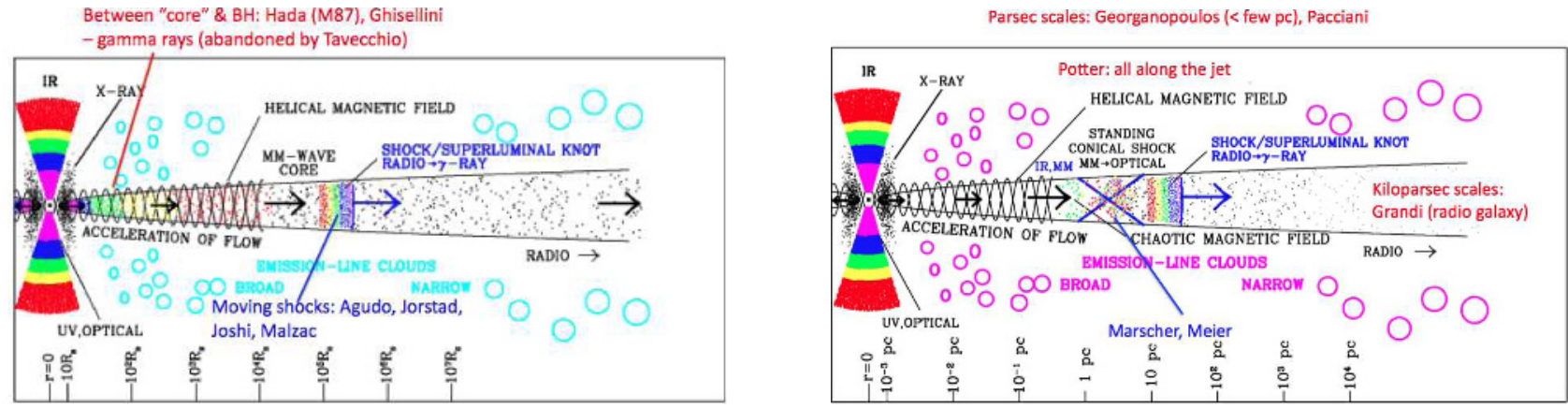

Figure 3. Locations where various attendees place the $\gamma$-ray outbursts. Where do you stand?

The problem of very short time-scales of variability is partly solved by the extremely narrow opening angle of the emission channel of a blazar jet. As stressed by Eric Clausen-Brown and Matt Lister, at any given time this angle is considerably less than $1^{\circ}$, even if the time-averaged jet (seen by stacking images from different epochs) is wider. This, combined with the possibility that the filling factor within these slivers of the jet might be small as well, can allow for the observed ultra-rapid variability. However, Gabriele Ghisellini pointed out that we should then expect that the time-scale of variability should be longer near the peaks of the SED, since the filling factor should be larger at those frequencies. Although variations at IR wavelengths, where the synchrotron SED peaks in quasarclass blazars, do generally have longer time-scales than optical variations, the possibility of such an effect should be investigated at $\gamma$-ray energies as well.

The limited sources of seed photons at distances of parsecs from the central engine is a serious issue, since even the molecular torus is unlikely to do the job beyond 2 or 3 parsecs. There may be plenty of synchrotron photons from slower portions of the jet, but this source has some trouble in reproducing the $\mathrm{X}$-ray to $\gamma$-ray SED. Another puzzle with TeV-emitting high-synchrotron-peak (HSP) BL Lac objects is that a high Doppler factor seems necessary to explain very rapid X-ray/TeV flares, but most such objects exhibit motion less than $\sim 2 c$ and relatively slow changes in flux, as found by Glenn Piner, Joseph Richards, and Matt Lister. Exceptions, however, include 3C 66A and $0716+714$, both of which exhibit highly superluminal motion. Maybe the problematic HSPs are just as fast but for some reason do not make knots on parsec scales whose motion can be followed and whose evolution leads to pronounced flux variations.

Numerical models of moving "internal" shocks in jets, which are still suspected of producing flares and superluminal knots, were presented by Manasvita Joshi, Xuhui Chen, Margo Aller, and Jesús Rueda Becerril. Jesus Rueda and Xuhui Chen stressed that magnetic fields in shocks need to be included in a physical way in shock models, while Julien Malzac proposed that multiple shocks can explain the flat spectra of XRB jets.

It is a scandal that prominent astrophysicists armed with comprehensive datasets disagree about where the gamma-ray flares occur and therefore on what the source of the seed photons is. Various presenters took various stands on the issue, as illustrated in the two cartoons in Figure 3. The resolution may be that flares can and do occur throughout the jet. In that case, we should see different properties for different flares, a prediction that has some observational support (see my review in these proceedings for a discussion). Furthermore, Joseph Richards reported that a major TeV flare in Mkn421 occurred 40 days before a rare radio flare, which indicates that the $\gamma$-ray event took place well upstream of the radio emission.

As usual for blazars, the situation is confusing. We therefore should admit to the possibility that all of our proposed solutions are wrong, in which case we need radical surgery on our paradigm.

\section{Objects with Low Accretion Power and $\mathrm{X}$-ray Binaries}

We can learn a lot about the overall jet phenomenon by adding objects with low accretion rates to our studies. In this vein, Sera Markoff noted that section A-B of the "Q" diagram of XRBs (Fig. 4) may be similar to low luminosity active galactic nuclei (LLAGN). During this hard state, an XRB produces a steady jet. Daniel Evans discussed how low-excitation radio galaxies have power-law X-ray spectra, with no sign of gas. Whatever powers jets also suppresses the X-ray Fe line emission, which he thinks might be explained by magnetically arrested accretion. As Juan Antonio Fernández Ontiveros also found from observations of the IR emission from LLAGN, jets are more dominant at very low accretion levels. A logical conclusion is that there should be naked AGN with $L / L_{\text {edd }}=0$ and nothing but jets! M87, which is the object of many studies attempting to understand what jets are like in general, seems to be very close to this extreme state. Carolina Casadio, Masanori Nakamura, Akihiro Doi, and Motoki Kino all presented interesting investigations of the properties of the M87 jet.

Simone Migliari reported the discovery of new SS433like XRB. We could use more, since SS433 has given us a lot of clues on the nature of XRBs. The "Q" diagram organizes spectral and jet characteristics, as reviewed by Sera Markoff, but there is a wide range of behavior, as emphasized by Fiamma Capitanio, Melania Del Santo, and Tariq 


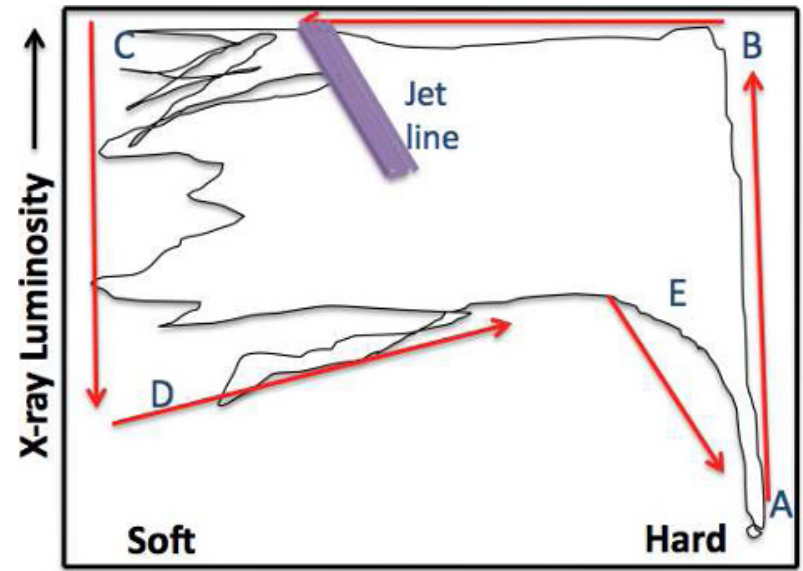

X-ray Spectral Hardness

Figure 4. Sketch of "Q" diagram of X-ray binaries. Lowluminosity, steady jets persist in the hard state (marked as E, A, and B) and as the X-ray spectrum becomes softer until the X-ray emission crosses the jet line. At this position on the diagram, a bright emission feature is ejected into the jet, after which the jet shuts off.

Shahbaz. So, we need to search for more, for example via the observing program described by Josep Martí. Virginia Cúnio has compiled a catalog of stellar-mass black hole binaries that facilitates comparison of the properties of the different systems.

\section{The (Un)importance of Black Hole Spin}

One of the major questions in the generation of jets is the conditions under which an accreting black hole system produces jets - and is therefore radio-loud - versus when it does not. Sasha Tchekhovskoy found in his simulations that near-maximal spin of the $\mathrm{BH}$ is needed for jets with the highest powers (relative to accretion powers). The spins need to be prograde, he reported. But Sera Markoff noted that XRB jets turn on and off, whereas the BH spin obviously cannot change so rapidly. This strongly suggests that spin is not main determining factor for making jets. It is not at all clear how this discrepancy can be resolved. Another scandal!

\section{The Importance of Polarization as a Probe of the Magnetic Field}

Since magnetic fields are mentioned in the title of the conference, many of the presentations discussed how we can used polarization observations to infer the geometry of the field in different regions of the jet. The degree of polarization measures the level of (dis)order of the magnetic field, although even if there are only two regions with nearly orthogonal field directions, we might mistakenly conclude that the field is chaotic. The electric-vector position angle (EVPA) indicates the mean direction of the magnetic field, projected on the sky, if optical depth and Faraday rotation are negligible. We can then compare the mean field direction relative to the orientation of the jet axis, bends, etc., all of which was covered in a review by John Wardle. As he mentioned, expected spacetime effects near the black hole can potentially be tested through VLBI observations at $\lambda \sim 1 \mathrm{~mm}$ by the Event Horizon Telescope.

As John Wardle discussed, circular polarization results from the basic synchrotron emission in an electron-proton plasma, in which case it is a few tenths of a percent at radio frequencies (roughly equal to the electron rest mass divided by the energy of an electron whose critical frequency equals the frequency of observation). Stronger circular polarization can be produced through partial conversion from linear to circular polarization as the radiation propagates through a plasma. Circular polarization at roughly the $1 \%$ level has been observed in a number of blazars, as presented by Hugh Aller, Shane O'Sullivan, and Denise Gabuzda. It is variable, as one would expect since blazar jets are highly dynamic, but the polarity can persist for longer time than one might expect.

Carlos Carrasco Gonzalez reported that linear polarization has proven quite useful to find synchrotron components in the jets of young stellar objects. David Russell and Helen Jermak discussed how linear polarization provides diagnostics for exploring the properties of XRBs and $\gamma$-ray bursts. Svetlana Jorstad was able to connect optical emission region of a quasar during an outburst with a superluminal knot via rotation of the linear polarization vector of both components at the same time. She then associated a $\gamma$-ray flare with the same component, since it coincided with the optical flare.

Rotations of the linear polarization vector are potentially extremely powerful indicators of the magnetic field geometry. New episodes of rotations in blazars were reported at the meeting by Svetlana Jorstad, Valeri Larionov, Monica Orienti, Pedro Paulo Beaklini, Erika Benítez, Sang-Sang Lee, and Mahito Sasada. As John Wardle and I summarized in our reviews, coherent rotations can be explained by an emission feature, such as a shock, following a spiral path in a helical magnetic field. However, it could instead be the body of the jet itself that twists, for example, because of a helical instability, as favored by Valeri Larionov to explain variations in the quasar CTA102 (and previously in $0716+714$ ). Alternatively, the apparent rotations can just be a manifestation of random walks when the magnetic field is turbulent. Or, a knot with a frozen-in magnetic field could simple rotate as it moves down the jet, as advocated in $0836+710$ by Svetlana Jorstad.

How can we tell the difference between these alternatives? Sebastian Kiehlman introduced a new technique for discerning between the turbulence explanation and other models, based on comparison of observed variations with the time behavior of the polarization from simulated sources with turbulent magnetic fields. Predictions of the other models for polarization rotations need to be worked out. Occasionally we get lucky: Sol Molina examined a time sequence of VLBA images of the favorably oriented quasar NRAO150, finding strong evidence for rotational motion in the jet, which can be explained by a helical twist of the body of the jet, in agreement with Valeri Larionov's model. 


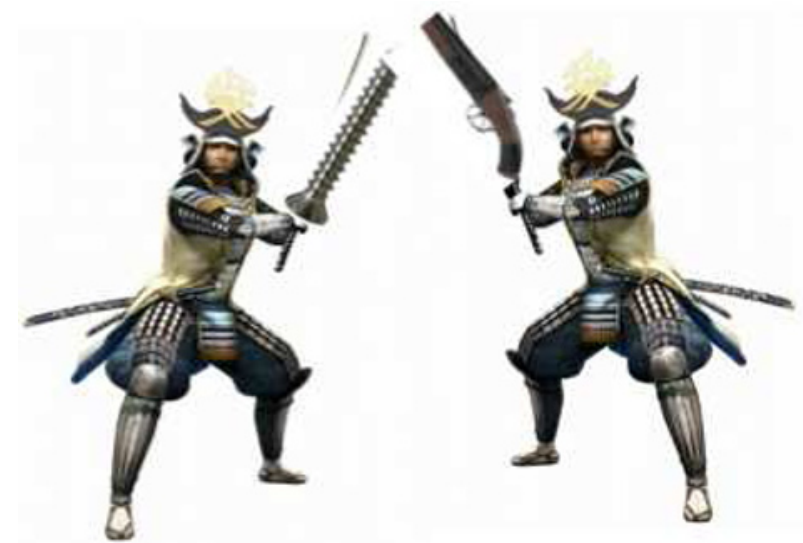

Figure 5. Samurais, representing Gabuzda and Marscher, dueling with, respectively, a helical spear and a shotgun (representing turbulence).

José Acosta Pulido presented near-IR polarimetric measurements at the meeting. We need more observations at this waveband in order to detect IR components that are not apparent at optical wavelengths, and also to measure wavelength dependence in the degree and position angle.

Another scandal exposed at the conference involves disagreement about the geometry of the magnetic field of jets on parsec scales. While apparently coherent rotations of polarization vectors have been used as evidence for the helical fields predicted by theorists closer to the black hole, there are reasons to expect a residual helical field, the chaos of turbulence, longitudinal fields stretched out by velocity shear, or either transverse or oblique fields from shock compression. The battle waged at the meeting, depicted in Figure 5, pitted helical (proponent: Denise Gabuzda) versus turbulent (which I defended) magnetic geometries. Points favoring helical fields include the need for this geometry to explain the acceleration and collimation of jets, combined with the finding that many jets continue to accelerate on parsec scales; the ability to explain rotations of polarization vectors observed in a number of blazars, as well as the kinks and wave-like structure reported by Marshall Cohen in BL Lacertae; and the prediction of Faraday rotation gradients across the jet, which have been measured by Denise Gabuzda, Shane O’Sullivan, Evgeniya Kravchenko, Eoin Murphy, and Mohammad Zamaninasab. But I counter that helical fields have a hard time explaining that the degree of linear polarization is usually both low and variable, and that the EVPA fluctuates, in bright blazars.

Favoring disordered fields are the low degrees of polarization, expecially in the VLBI cores; variations in degree of polarization and EVPA; the expectation that current-driven instabilities (e.g., the kink instability discussed by Nektarios Vlahakis, Ken-Ichi Nishikawa, and Yosuke Mizuno) should convert a helical field into magnetic turbulence as the kinetic energy approaches the magnetic energy; the ability of turbulence to produce rapid fluctuations in flux density, major flares, and power-law power spectra; and the ability of shocks that compress a disordered magnetic field to explain polarization events seen at radio wavelengths, as presented by Margo Aller. But I have to admit that this model fails to explain Faraday rotation gradients across jet (perhaps this could be in the sheath, though).

Who won the battle? Neither side - it rages on! Actually, the battle was rather quiescent, and we agreed at the end that the truth might lie somewhere in neutral territory, with a turbulent field superposed on a helical component. I intend to put this geometry into my TEMZ code in order to see whether the simulated polarization variations resemble those that are observed.

\section{Hadrons vs. Leptons}

Our knowledge of the positively charged particles inside jets is quite limited. Positrons might greatly outnumber protons, or vice versa. If protons dominate, do they hold $\sim 100$ times the energy density as electrons, are they in equipartition, or do they even have less energy than the electrons? In his review, Markus Böttcher reported that the SEDs of blazars can be fit as well by hadronic models as by leptonic models. There is a high cost, though: a factor of $\sim 100$ in energy. This seems too much if blazars have an energy crisis, but could be reasonable in objects with high accretion rates. And the factor of $\sim 100$ is similar to the ratio of the proton to electron energy densities in Galactic cosmic-rays. On the other hand, Apostolos Mastichiadis and Maria Petropoulou expressed difficulty in their efforts to produce light curves similar to PKS 2155-304 with a hadronic model, although they will continue to try.

\section{New Activity in Familiar AGN}

A number of presentations demonstrated that many of our old friends are still very much in vogue. Among these, Mkn421 remains everyone's favorite $\mathrm{TeV}$ blazar, with Reshmi Mukherjee, Joseph Richards, Rocco Lico, Mislav Balokovic, Kotaro Niinuma, Apostolos Mastichiadis, and Elena Racero all reporting new observations or modeling of the BL Lac object. The radio galaxy $3 \mathrm{C} 84$ is making a comeback, having been detected as a $\gamma$-ray source by Fermi and rejuvenating its radio emission by changing the direction of its jet, as reported by Hiroshi Nagai. Antxon Alberdi presented evidence that the jet of the relatively nearby modestly-active LLAGN of the spiral galaxy M81 shows signs of precession. Tobias Beuchert presented a long-term study of the radio galaxy $3 \mathrm{C} 111$, which teeters on the edge of blazardom with its superluminal motion and explosive flux changes. And the classic BL Lac object OJ287 decided to launch itself back into the limelight by changing the direction of its jet by more than $90^{\circ}$ and resuming its rapid-fire outbursts, as studied by Margo Aller, Iván Agudo, and Satoko Sawada-Satoh.

\section{Advancements in Observations and Theory}

We are truly in the midst of a golden age of exploration of relativistic jets. We are amassing light curves, po- 
larization vs. time curves, and SEDs with unprecedented time and frequency coverage. Presentations displaying the rich datasets included those by Sarah Kaufmann, Valeri Larionov, Darya Morozova, Ivan Troitskiy, Elena Racero, Claudia Raiteri, Immacolata Donnarumma, and me. Vladimir Hagen-Thorn also presented light curves, to which he applied his robust method for separating constant and variable component spectra, which I recommend to everyone who possesses simultaneous multi-wavelength, multi-epoch flux measurements. Talviki Hovatta put radio flux measurements to good use by deriving variability Doppler factors for many blazars after applying a new Bayesian Markov Chain Monte Carlo method to fit the light curves with multiple distinct flares. This allows a study of the intrinsic brightness temperatures of large samples of blazars.

A number of attendees, including Manasvita Joshi, Xuhui Chen, Stephan Richter, Yaxk'in U Kan Coronado, Maria Petropoulou, William Potter (whose model involves a parabolic jet, a geometry that I employed in my first paper on jets, in 1980 - déjà vu!), Jesús Rueda Becerril, Marco Sorcia, Thomas Vuillaume, and Matthias Weidinger, have developed computational codes that try to simulate time-dependent SEDs and light curves of blazars. My own TEMZ code adds linear polarization vs. time to this. None of these has yet succeeded in re-creating blazar behavior faithfully, but it is encouraging that the range of scenarios is expanding.

\section{Very Interesting . . .}

As occurs at every meeting, there were a number of results that were interesting enough for me to take notice and list separately:

- Markos Georganopoulos analyzed the SED of the kiloparsec-scale jet of 3C 273 and concluded that the Xray emission is from synchrotron radiation by electrons with energies in the $\mathrm{TeV}$ range. - Cornelia Müller analyzed a very unusual $\gamma$-ray source PMN J1603-4904, which has VLBI structure like a compact symmetric object, an IR hump like a starburst, bright and steady gamma-ray emission, and maybe a BL Lac type optical spectrum. I will be very curious to learn what further observations will reveal about this oddball object.

- Anabela Teresa Araudo pointed out that supergiant stars are plentiful in galactic nuclei. Their winds can enter a jet, possibly causing detectable gamma-ray emission.

- Matt Lister, Marcello Giroletti, Monica Orienti, and Glenn Piner concluded from their studies that gamma-ray and radio emission grow up together. So, they are siblings with the jet as the parent.

- GeV $\gamma$-ray and optical emission are usually — but not always (Xuhui Chen) — the best of friends, according to results presented by Maria Isabel Carnerero Martin, Svetlana Jorstad, Valeri Larionov, Ivan Troitskiy, and others. - Eileen Meyer found that the existence of highly beamed "fake BL Lacs" would help to explain the blazar sequence, the relation between the luminosities and SEDs of blazars. - There are also blazars disguised as steep-spectrum radio sources (Eleonora Torresi), bright double-lobed sources (Dave Hough), and narrow-line Seyfert 1 galaxies (Filippo D'Ammando and Annika Kreikenbohm).

- Ulf Menzler considered plasma effects on the behavior of secondary particles made by $\gamma$-rays traversing intergalactic space, finding that the expected spectrum of inverse Compton radiation off the Cosmic Microwave Background is modified during propagation. This affects the derivation of the intergalactic magnetic field from observations of the radiation.

\section{Progress Is Being Made}

Even as some of our favorite observing facilities are being closed down, or threatened with closure (see below), advances in the field continue. Yuri Y. Kovalev reported that the Russian RadioAstron VLBI satellite has been deployed and is working well, so we should soon be seeing visibilities and images with unprecedented resolution at frequencies up to $22 \mathrm{GHz}$. Masanori Nakamura discussed observations of M87 with $\lambda 1 \mathrm{~mm}$ VLBI, which can potentially image emission around the event horizon. Colm Coughlan and Juliana Motter are applying maximum-entropy and cross-entropy methods to analyze VLBI data. Particular effort is going into refinement of measurements of core shifts from opacity effects in order to derive some physical conditions, as detailed by Kazuhiro Hada, Takafumi Haga, Denise Gabuzda, Alexander Kutkin, and Pëtr Voytsik. Florent Mertens explained a new technique that he has developed with Andrei Lobanov to measure both longitudinal and transverse velocity gradients in jet flows.

\section{The Future}

As Yoda said in the movie The Empire Strikes Back, "Difficult to see is the future, always in motion it is." As in that movie, we are in dark times, when the evil empire of nonVLBI observers in the US has been cutting funds from two of our most valuable instruments, Fermi and the VLBA. Yes, dark energy and Earth-like exoplanets are important and interesting, but the public will be very disappointed if we curtail research on black-hole systems!

There are, though, some bright lights on the horizon. One is already beyond the horizon: RadioAstron is taking VLBI way beyond Earth-diameter baselines and to frequencies where no VLBI antenna has ever gone before. And Gabriele Giovannini told us about a program to obtain ultra-high resolution images of jets through VLBI observations of large samples of $\gamma$-ray detected blazars.

ALMA, whose completion was just announced at this writing, promises to bring us SEDs, light curves, and polarization of blazars at submillimeter wavelengths if we can manage to get observing time, as stressed by Kazuhiro Hada and Daniel Evans. I hope that the heavy demands on ALMA won't prevent us from securing time coverage dense enough to carry out the studies we desire of jets at this important waveband.

As promoted by Reshmi Mukherjee, Manel Errando, Sarah Kaufmann, and Marco Sorcia, TeV observations 
with the planned Cherenkov Telescope Array will provide us with higher sensitivity and a lower energy threshold for $\gamma$-ray observations. This will be extremely important in the post-Fermi era. But we need to lobby our fellow astronomers and the fundings agencies to keep other prime time domain instruments operating.

On the theoretical front, self-consistent simulations of jet physics and emission should soon be possible. One such effort is being organized by Ken-Ichi Nishikawa. Per-

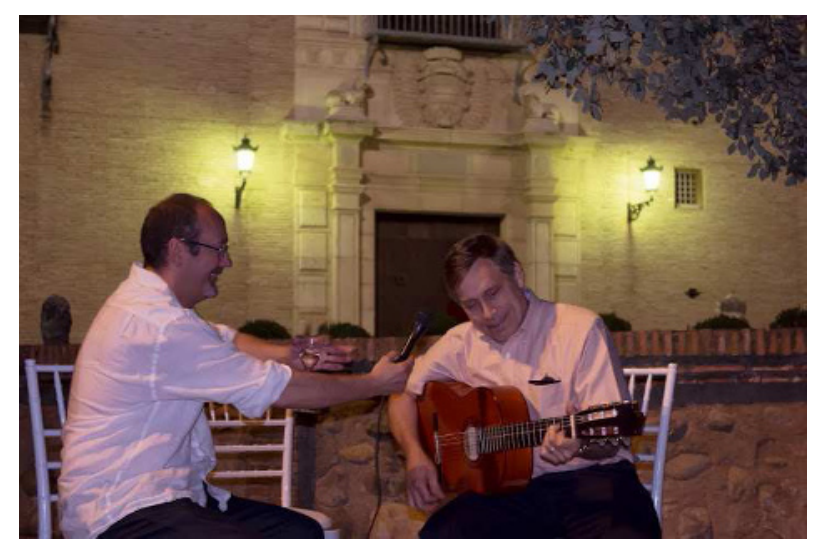

Figure 6. Scene from the conference banquet, al fresco overlooking Alhambra: Marscher singing his composition "Superluminal Lover" with the conference host, José Luis Gómez, holding the microphone. [Photo by Satoko Sawada-Satoh]

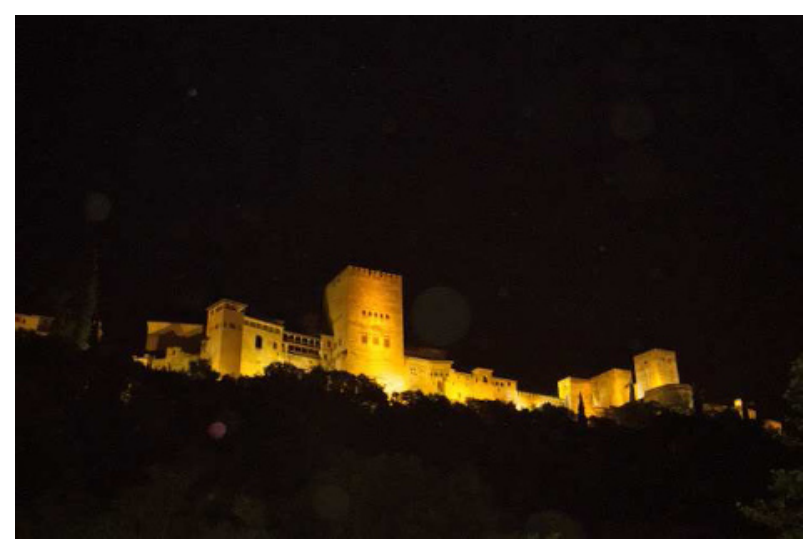

Figure 7. Overlooking the site of the conference banquet is the famous Alhambra, which conference attendees visited during a free afternoon. [Photo by Satoko Sawada-Satoh] haps someday this will advance to the level where we can claim to have a JEt Simulation Telescope (JEST)! And I have just the project for it, inspired by the conference: Jet Observations through Simulation Experiments: Light curves, Undulations, Images, and SEDs

Let's see, what would be the acronym for this? . . JOSÉ LUIS! Muchas gracias, amigo — you are a real superluminal lover (see Fig. 6) and you organized a fantastic meeting!! 